\title{
Adsorption and absorption of boron, nitrogen, aluminum, and phosphorus on silicene: Stability and electronic and phonon properties
}

\author{
J. Sivek, ${ }^{*}$ H. Sahin, ${ }^{\dagger}$ B. Partoens,${ }^{\ddagger}$ and F. M. Peeters ${ }^{\S}$ \\ Departement Fysica, Universiteit Antwerpen, Groenenborgerlaan 171, B-2020 Antwerpen, Belgium
}

(Received 1 October 2012; published 27 February 2013)

\begin{abstract}
$A b$ initio calculations within the density-functional theory formalism are performed to investigate the chemical functionalization of a graphene-like monolayer of silicon-silicene-with $\mathrm{B}, \mathrm{N}, \mathrm{Al}$, or P atoms. The structural, electronic, magnetic, and vibrational properties are reported. The most preferable adsorption sites are found to be valley, bridge, valley and hill sites for $\mathrm{B}, \mathrm{N}, \mathrm{Al}$, and $\mathrm{P}$ adatoms, respectively. All the relaxed systems with adsorbed/substituted atoms exhibit metallic behavior with strongly bonded $\mathrm{B}, \mathrm{N}, \mathrm{Al}$, and $\mathrm{P}$ atoms accompanied by an appreciable electron transfer from silicene to the $\mathrm{B}, \mathrm{N}$, and $\mathrm{P}$ adatom/substituent. The $\mathrm{Al}$ atoms exhibit opposite charge transfer, with $n$-type doping of silicene and weaker bonding. The adatoms/substituents induce characteristic branches in the phonon spectrum of silicene, which can be probed by Raman measurements. Using molecular dynamics, we found that the systems under study are stable up to at least $T=500 \mathrm{~K}$. Our results demonstrate that silicene has a very reactive and functionalizable surface.
\end{abstract}

PACS number(s): 61.72.uf, 73.22.-f, 68.43.Fg, 73.20.Hb

\section{INTRODUCTION}

Since the first reports on the successful isolation of a stable monolayer of graphene ${ }^{1,2}$ special efforts have been invested in the exploration of similar materials with novel properties resulting from their ultrathin two-dimensional nature. Those alternative materials have the potential to bypass some of the obstacles existing in the usage of graphene in contemporary electronics, i.e., incompatibility with present day silicon technology and lack of an energy band gap, which is essential for all semiconductor devices. For graphene itself, further modification is inevitable in order to tailor its electronic properties. A broad range of chemical decoration ${ }^{3-7}$ and substitutional doping ${ }^{8-10}$ have been investigated to open and tune the band gap of graphene. Particular attention was given to boron and nitrogen as the first choice elements because of their chemical propinquity to carbon as well as to silicon. ${ }^{11}$ The second row elements have not been omitted, $\mathrm{Al}, \mathrm{Si}$, and $\mathrm{P}$ doping induce band-gap opening in monolayer graphene. ${ }^{12}$ Theoretical studies of functionalization of graphene have later been supported by the experimental realization of substitutional doping with $\mathrm{B}$ and $\mathrm{N}$ atoms via arc discharge using graphite electrodes in the presence of hydrogen and $\mathrm{B}$ or $\mathrm{N}$ atom incorporating molecules (pyridine, amonia, $\left.\mathrm{B}_{2} \mathrm{H}_{6}\right){ }^{8}$ thermal annealing of graphene in the presence of boron oxide ${ }^{9}$ or nitrogen plasma treatment of graphene. ${ }^{10}$

Recently, the monolayer honeycomb structure of silicon, silicene, has emerged as a potential few-atom-thick material to replace graphene. As was reported by early theoretical works, silicene is a semimetal with linearly crossing bands and a zero electronic band gap similar to graphene. ${ }^{13-15}$ Furthermore, similar to graphene, electrons propagating through the monolayer crystal structure of silicene are predicted to show massless fermion behavior in the vicinity of the Dirac point. Additionally, some unique features of monolayer silicene such as quantum spin Hall effect, ${ }^{16}$ a large spin-orbit interaction, ${ }^{17}$ a mechanically tunable band gap ${ }^{18}$ and a valley-polarized metallic phase $^{19}$ have been reported by theoretical studies. Moreover, the recent experimental observations and synthesis of silicene $\mathrm{e}^{20-24}$ have opened a new path for nanoscale materials that might be easily functionalized chemically or mechanically and incorporated within electronics as we know it today. ${ }^{25}$ Very recently, we reported the adsorption characteristics of alkali, alkaline-earth, and transition-metal atoms on monolayer silicene. ${ }^{26}$ Differing from graphene, one can expect the substitution of $\mathrm{B}, \mathrm{N}, \mathrm{Al}$, or $\mathrm{P}$ atoms to be more likely on the silicene surface [see Fig. 1(a)], as it is more reactive due to its $s p^{3}$-like lattice structure.

In this paper, motivated by the route undertaken with graphene and the recent synthesis of silicene, we investigate the adsorption and absorption of $\mathrm{B}, \mathrm{N}, \mathrm{Al}$, and $\mathrm{P}$ atoms on the surface of pristine free-standing silicene together with their substitutional doping. Our interest is pointed towards the compounds' structure, binding energies of the most stable structures and their electronic, magnetic, and phonon properties. We find that the adsorbed $\mathrm{B}, \mathrm{N}$, and $\mathrm{P}$ atoms are strongly bonded to the underlying silicene, attached to its surface but also penetrated into the silicon lattice. Weaker bonding and immersion is observed for the $\mathrm{Al}$ atom. These observations differ from the general chemical functionalization of graphene, which is highly dependent on the presence of defects and crystal boundaries. ${ }^{27}$ The stability of the ground state structures has also been addressed by using ab initio molecular dynamics and phonon calculations.

\section{COMPUTATIONAL METHODOLOGY}

Our present investigation of the chemical modification of silicene employs density functional theory (DFT) as implemented in the VASP package. ${ }^{28}$ We have made use of the spin-polarized local-density approximation (LDA) ${ }^{29}$ for the exchange-correlation functional, the projector augmented wave method ${ }^{30}$ and a plane-wave basis set with an energy cutoff of $500 \mathrm{eV}$. The sampling of the Brillouin zone was done for the supercell with the equivalent of a $24 \times 24 \times 1$ Monkhorst-Pack $k$-point grid for a silicene unit cell (containing two silicon atoms). The partial occupancies in the electronic ground-state calculation were treated using the tetrahedron methodology with Blöchl corrections ${ }^{31}$. For the purpose of the 
calculation of the density of states, a Gaussian smearing of the energy levels was applied with standard deviation set to $0.1 \mathrm{eV}$.

To eliminate the interaction emerging from periodic boundary conditions in all three dimensions, a $4 \times 4$ supercell was used with the height of $15 \AA$ to include enough vacuum, and dipole corrections were used. All reported quantitative results of the charge transfer were obtained by usage of the Bader charge population analysis, ${ }^{32,33}$ and the iterative modified Hirshfeld charge population analysis. ${ }^{34,35}$

The relaxation of the atomic positions was performed with forces smaller than $0.01 \mathrm{eV}^{-1}$. To reduce the strain induced by the adsorbates/substituents, the lattice parameters were optimized properly. The phonon frequencies for adatom adsorbed/substituted silicene were calculated using the small displacement method. ${ }^{36}$

$A b$ initio molecular dynamics simulations (MD) were performed with use of the nonspin polarized local-density approximation (LDA) ${ }^{29}$ for the exchange-correlation functional, the projector augmented wave method ${ }^{30}$ and a plane-wave basis set with an energy cutoff of $500 \mathrm{eV}$. The sampling of the Brillouin zone was done for the supercell with the equivalent of a $12 \times 12 \times 1$ Monkhorst-Pack $k$-point grid for a silicene unit cell. The integration of Newton's equations of motion was performed using the Verlet algorithm where Harris corrections were used in order to correct the forces. The simulations were performed within the microcanonical (NVE) ensemble with velocities assigned according to the Maxwell-Boltzmann distribution at the temperature of $500 \mathrm{~K}$ during the entire (a)

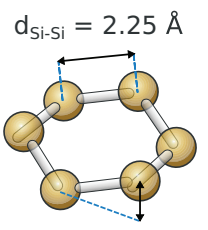

$\delta=0.44 \AA$

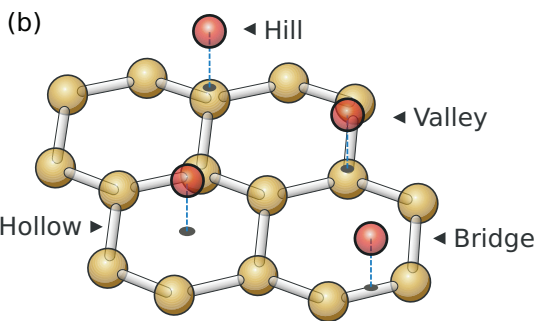

(c)

Silicene

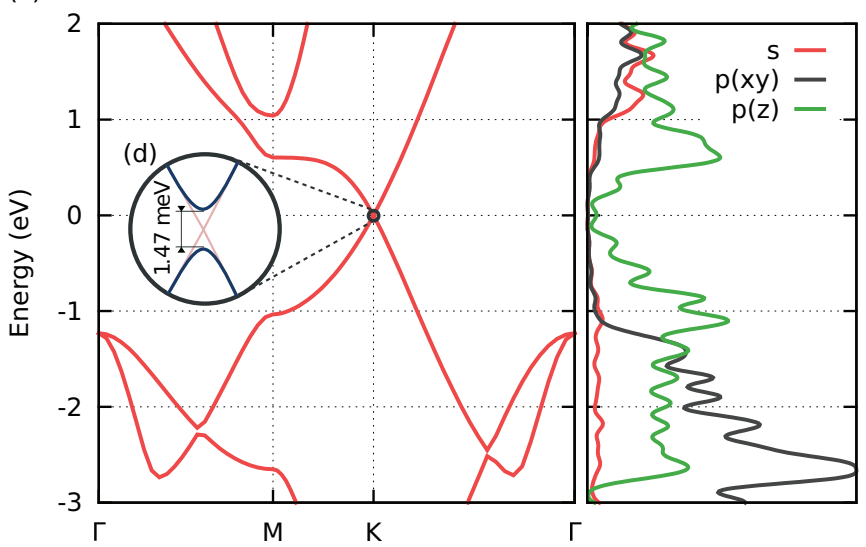

FIG. 1. (Color online) (a) Structural parameters for silicene and (b) possible adsorption sites on the silicene lattice. (c) Electronic band dispersion and partial density of states for perfect silicene. The energies are relative to the Fermi level (i.e., $E_{\mathrm{F}}=0$ ). The inset (d) displays the calculated spin-orbit gap of $1.47 \mathrm{meV}$. calculation. To avoid large temperature fluctuations, velocities were normalized every 40 steps. The total duration of the simulation was 2 ps with the time step equal to $1 \mathrm{fs}$.

\section{RESULTS AND DISCUSSION}

\section{A. Atomic structure and migration barriers}

First, we consider the adsorption of a single atom on a silicene surface. In contrast to the completely flat one-atomthick surface of graphene, silicene is buckled as can be seen in Fig. 1(a) and we can expect higher reactivity due to this $s p^{3}$-like lattice structure. Similar to graphene, silicene is a semimetal with linearly crossing bands at the Fermi level with a zero electronic band gap [see Fig. 1(c)]. However, the effect of spin-orbit coupling, that yields $1.47 \mathrm{meV}$ spin-orbit gap [see Fig. 1(d)], is much larger than that of graphene's. ${ }^{37} \mathrm{We}$ define the binding energy for adsorption as $E_{\mathrm{B}}=E_{\text {system }}-$ $\left(E_{\text {silicene }}+E_{\text {adatom }}\right)$. There are three possible adsorption sites on graphene, while as a consequence of the buckled hexagonal lattice structure, silicene has now four different adsorption sites as shown in Fig. 1(b): above the center of the hexagonal silicon rings (hollow site), on top of the upper silicon atoms (hill site), on top of the lower silicon atoms (valley site), and on top of the $\mathrm{Si}-\mathrm{Si}$ bond (bridge site).

The possible diffusion paths were determined from the energetics of the adatoms on the different lattice points. As shown in Fig. 2, each $\mathrm{N}$ adsorbate has to overcome an energy barrier of $\sim 0.7 \mathrm{eV}$ for diffusion from one bridge site to a neighboring one via a valley site. $\mathrm{B}$ adsorbates, because of their stronger binding energy compared to $\mathrm{N}$ atoms, see larger migration barriers. Diffusion of a B atom from one valley site to another one can occur via a hollow site by overcoming the energy barrier of $\sim 2.0 \mathrm{eV}$. The $\mathrm{P}$ adatom has to overcome an energy barrier of $\sim 0.9 \mathrm{eV}$ when diffusing between neighboring hill sites via the bridge and valley sites. For the $\mathrm{Al}$ adatoms the energy barrier is $\sim 0.4 \mathrm{eV}$ between two neighboring valley sites. After unconstrained relaxation, the four adsorption sites

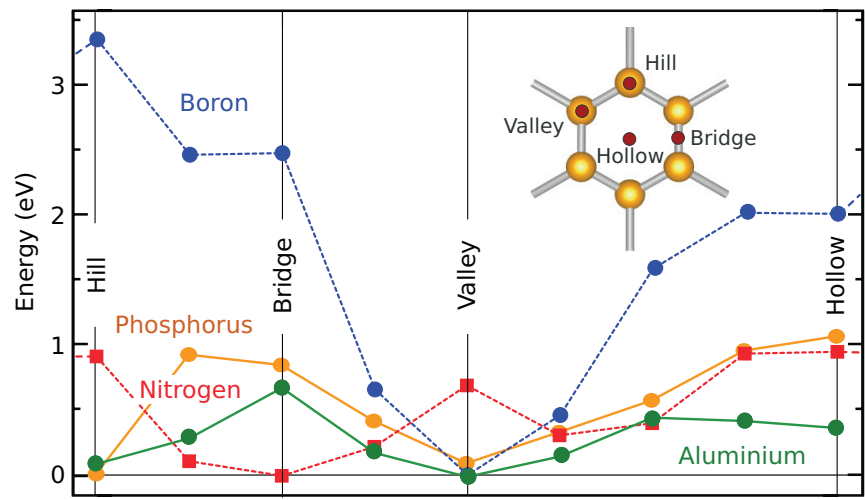

FIG. 2. (Color online) Diffusion characteristics of $\mathrm{B}, \mathrm{N}, \mathrm{Al}$, and $\mathrm{P}$ adsorbates. The energies are relative to the binding energy of the most preferable adsorption site. Spatial distance is plotted on $x$ axis, the intermediate positions between high-symmetry points were obtained by restricting the adatoms movement perpendicular to the silicene surface. The local minima in diffusion energy plots outside of the four high symmetry adsorption sites do not provide stable binding without aforementioned restrictions. 
TABLE I. Calculated parameters for $\mathrm{B}, \mathrm{N}, \mathrm{Al}$, and $\mathrm{P}$ adsorbed/substituted silicene: lattice constant $a$, adsorbate-Si bond distance $d_{\mathrm{SiX}}$, average distance to low-lying $\mathrm{Si}$ layer $h$, magnetic moment $\mu$, binding energy per adatom/substituent $E_{\mathrm{B}}$, work function $\Phi$ (for the adsorption the value corresponds to the value measured from the side of the adsorbate), charge located on adatom/substituent obtained with Bader charge analysis, $\rho^{\mathrm{B}}$, or iterative Hirshfeld method $\rho^{\mathrm{H}}$ and dipole moment $p$. The adsorbate and substituent concentration was one to 32 and $31 \mathrm{Si}$ atoms, respectively.

\begin{tabular}{|c|c|c|c|c|c|c|c|c|c|c|}
\hline & Site & $a(\AA)$ & $d_{\mathrm{SiX}}(\AA)$ & $h(\AA)$ & $\mu\left(\mu_{\mathrm{B}}\right)$ & $E_{\mathrm{B}}(\mathrm{eV})$ & $\Phi(\mathrm{eV})$ & $\rho^{\mathrm{B}}(\mathrm{e})$ & $\rho^{\mathrm{H}}(\mathrm{e})$ & $p(\mathrm{e} \AA)$ \\
\hline B (ads) & Valley & 15.16 & 1.95 & 0.71 & 0.0 & -5.85 & 4.73 & -1.6 & -0.7 & -0.02 \\
\hline $\mathrm{N}$ (ads) & Bridge & 15.30 & 1.63 & 1.42 & 0.0 & -5.54 & 5.02 & -2.0 & -0.6 & 0.06 \\
\hline Al (ads) & Valley & 15.26 & 2.51 & 1.71 & 0.0 & -2.87 & 4.66 & 1.0 & 0.0 & 0.12 \\
\hline $\mathrm{P}(\mathrm{ads})$ & Hill & 15.19 & 2.33 & 1.18 & 0.5 & -5.28 & 4.89 & -0.9 & -0.2 & -0.16 \\
\hline B (sub) & N/A & 15.21 & 1.93 & 0.00 & 0.0 & -6.21 & 5.15 & -1.4 & -0.6 & -0.08 \\
\hline $\mathrm{N}$ (sub) & N/A & 15.03 & 1.80 & 0.14 & 0.9 & -5.64 & 4.67 & -2.1 & -0.4 & -0.03 \\
\hline $\mathrm{Al}(\mathrm{sub})$ & N/A & 15.43 & 2.36 & 0.44 & 0.0 & -2.28 & 5.04 & 1.6 & 0.3 & -0.03 \\
\hline $\mathrm{P}(\mathrm{sub})$ & N/A & 15.24 & 2.24 & 0.47 & 0.0 & -4.84 & 4.55 & -1.2 & -0.1 & 0.02 \\
\hline
\end{tabular}

provide stable or metastable binding, albeit with different binding energy. Only the adsorption of an $\mathrm{Al}$ atom on a bridge site is found to be unstable.

For the graphene surface, the bridge site was found to be the most favorable adsorption site for both $\mathrm{B}$ and $\mathrm{N}$ adsorbates. ${ }^{38}$ However, we find that the most favorable adsorption sites on the silicene surface are the valley and bridge sites for B and $\mathrm{N}$ adsorbates, respectively. While the adsorption of the $\mathrm{B}(\mathrm{N})$ atom occurs with a $-1.8(-4.6) \mathrm{eV}$ binding energy on graphene, ${ }^{38}$ for silicene the bonding between $\mathrm{B}(\mathrm{N})$ and the silicene lattice is stronger with a binding energy of -5.85 $(-5.54) \mathrm{eV}$. The $\mathrm{Al}$ and $\mathrm{P}$ atoms preferential adsorption sites were found to be the valley site with a binding energy of $-2.87 \mathrm{eV}$ and the hill site with an adsorption energy of $-5.28 \mathrm{eV}$, respectively. We found the adatoms to considerably distort the underlying silicene layer. A B adsorbate is almost completely immersed into the silicene layer and pushes the underlying $\mathrm{Si}$ atom down from its original position. The $\mathrm{B}$ adatom average distance from the underlying Si layer is only $0.71 \AA$ as can be seen in Table I. $\mathrm{N}$ atom adsorption on the bridge site results in $\mathrm{Si}-\mathrm{Si}$ bond breakage. A similar effect as with the $\mathrm{B}$ adsorption occurs for $\mathrm{P}$ and $\mathrm{Al}$ adatoms, albeit with lower intensity and with up to two times larger average distances from the underlying Si layer as can be seen in Table I.

Next, we investigate the absorption, i.e., the substitution of a single $\mathrm{Si}$ atom with a $\mathrm{B}, \mathrm{N}, \mathrm{Al}$, or $\mathrm{P}$ atom. The binding energies for substitutional doping are $-6.21,-5.64,-2.28$, and $-4.84 \mathrm{eV}$, for $\mathrm{B}, \mathrm{N}, \mathrm{Al}$, and $\mathrm{P}$ atoms, respectively. This binding energy for substutution is calculated as follows: $E_{\mathrm{B}}=E_{\text {system }}-\left[E_{\text {silicene }}\left(N_{\mathrm{Si}}-1\right) / N_{\mathrm{Si}}+E_{\text {adatom }}\right]$. B-Si and $\mathrm{N}-\mathrm{Si}$ bonds are shorter than the intrinsic $\mathrm{Si}-\mathrm{Si}$ bond lengths, the P-Si bond is also slightly shorter but the Al-Si bond length is larger by $0.1 \AA$ in comparison to the $\mathrm{Si}-\mathrm{Si}$ bond length as can be seen in Table I (comparabable to $\mathrm{N}$ and $\mathrm{B}$ substituted graphene ${ }^{8}$ ). The $\mathrm{B}, \mathrm{N}$, and $\mathrm{Al}$ substituent atom bonds with neighboring $\mathrm{Si}$ atoms are more flat, reducing local buckling. The $\mathrm{P}$ substituent atom exhibits comparable buckling as the $\mathrm{Si}$ atoms, the angle $\theta_{\mathrm{SiPSi}}\left(111.3^{\circ}\right)$ is even smaller compared to $\theta_{\mathrm{SiSiSi}}\left(116.3^{\circ}\right)$ of pure silicene. With increased distance from the $\mathrm{B}, \mathrm{N}, \mathrm{Al}$, or $\mathrm{P}$ atom, the buckling of silicene is quickly restored. All the substituents except B atom exhibit a small displacement from the containing Si layer.
As already mentioned, the $\mathrm{B}$ atom adsorption on the valley site (to some degree $\mathrm{P}$ atom adsorption on the hill as well) results in structures resembling substitution structures, with the $\mathrm{Si}$ atom pushed out of the silicene plane and adsorbate immersion into the plane. This can be a potential route for chemical doping of silicene via chemical decoration.

The calculated values for the binding energies show that substitutional doping is energetically more favorable for $\mathrm{B}$ and $\mathrm{N}$ atoms, whereas it is less favorable for $\mathrm{Al}$ and $\mathrm{P}$ atoms compared to adsorption. This behavior of the binding energy causes a lower amount of immersion for the $\mathrm{Al}$ and $\mathrm{P}$ atoms as compared to the $\mathrm{B}$ and $\mathrm{N}$ atoms.

We find the adsorbed as well as substituent $\mathrm{Al}$ atom to have notable lower absolute value for its binding energy in comparison to the other investigated atoms. The reason for this different behavior can be explained from a chemical point of view. The $\mathrm{Al}$ atom is the only one, due to its lower electronegativity compared to the $\mathrm{Si}$ atom that looses electrons in favor of the neighboring $\mathrm{Si}$ atoms. The induced depopulation of bonding orbitals increases the length of the $\mathrm{Si}-\mathrm{Al}$ bond $d_{\mathrm{SiAl}}$ and weakens it.

\section{B. Electronic structure}

In Table $\mathrm{I}$, the electronic properties of $\mathrm{B}, \mathrm{N}, \mathrm{Al}$, or $\mathrm{P}$ adsorbed silicene are also presented. Both $\mathrm{B}$ and $\mathrm{Al}$ atoms adsorbed on the valley site as well as the $\mathrm{N}$ atom adsorbed on the bridge site turn the semimetallic silicene into a nonmagnetic metal. Adsorption of $\mathrm{P}$ atom on the hill site introduces metallicity with a net magnetic moment of $0.5 \mu_{\mathrm{B}}$. The work function (measured at the side of the adsorbate) is slightly lowered to $4.73 \mathrm{eV}$ for $\mathrm{B}$ and $4.66 \mathrm{eV}$ for $\mathrm{Al}$ adsorption from $4.77 \mathrm{eV}$ for intrinsic silicene. On the other hand, adsorption of a $\mathrm{N}$ or $\mathrm{P}$ atom increases the work function to 5.02 or $4.89 \mathrm{eV}$, respectively.

We also present the partial density of states analysis for B, N, Al, and P in Figs. 3(a)-3(d). For B adsorption, the metallic bands crossing the Fermi level are formed by the hybridization of the $\mathrm{B}-p_{x y}$ peaks with the $\mathrm{Si}-p_{x y}$ and the $\mathrm{Si}-p_{z}$ states. However, since the adsorption of $\mathrm{N}$ occurs on top of a $\mathrm{Si}-\mathrm{Si}$ bond, the mixing of $\mathrm{N}$ states with the silicene states is more complex. It appears from Fig. 3(b) that while the main 


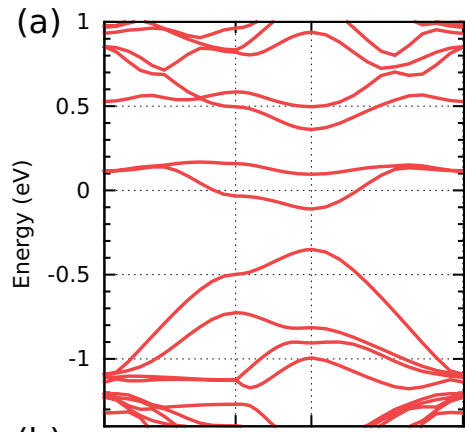

(b)
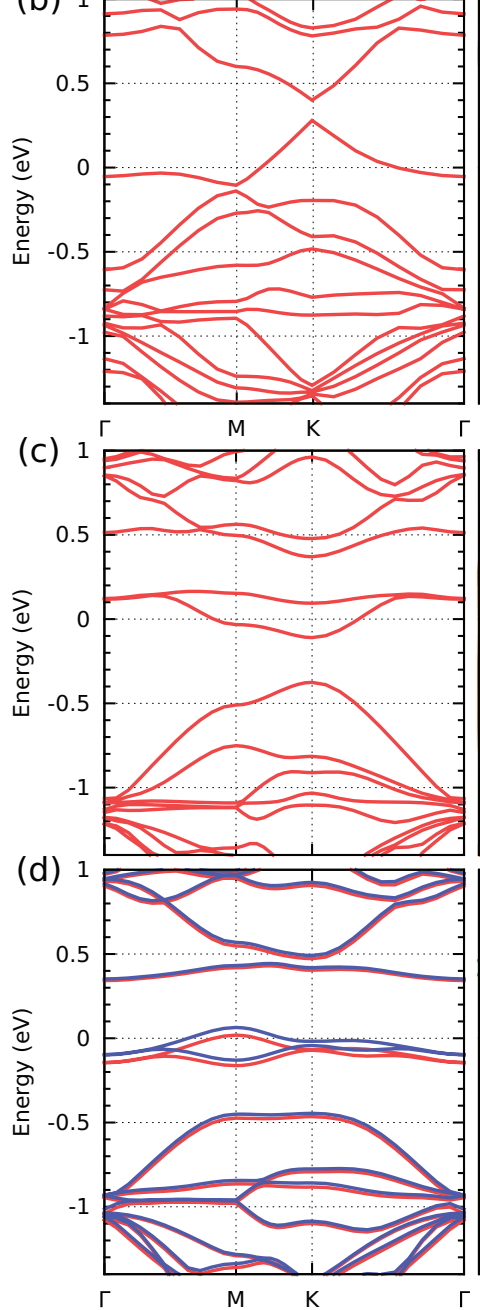
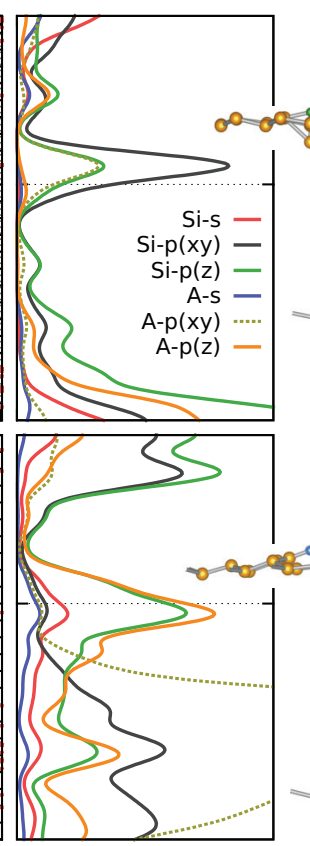

$\Gamma$

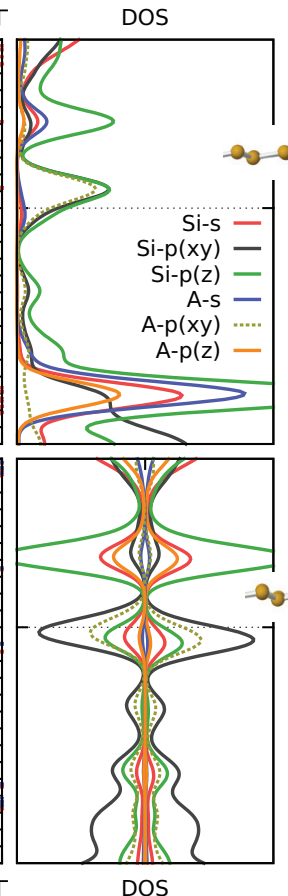

B adsorption

$\cos _{0-0}$ ald

B substitution -

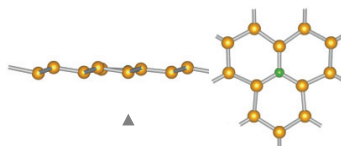

(f) 1

$4 \mathrm{~N}$ adsorption

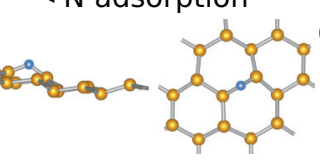

N substitution -

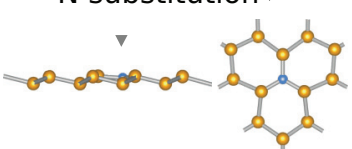

4 Al adsorption
(g)
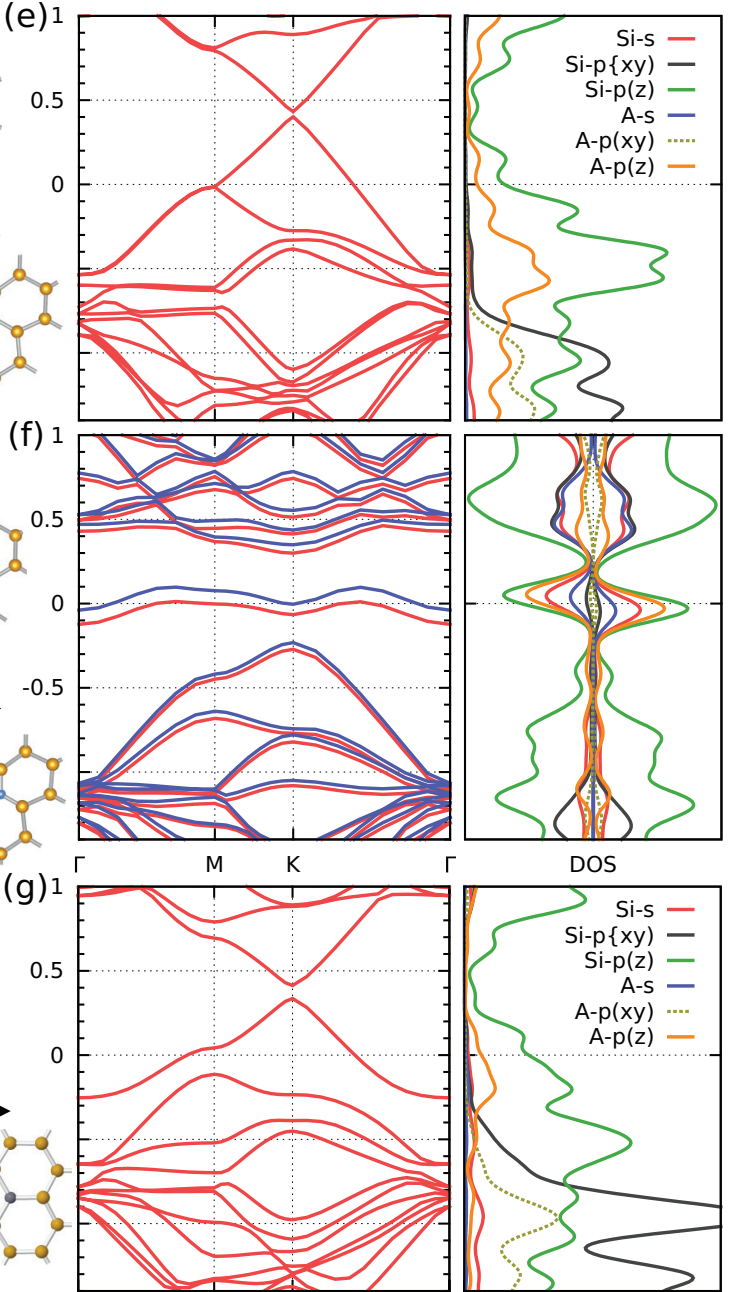

$4 \mathrm{P}$ adsorption

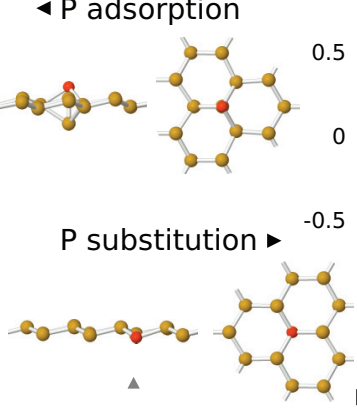

(h)

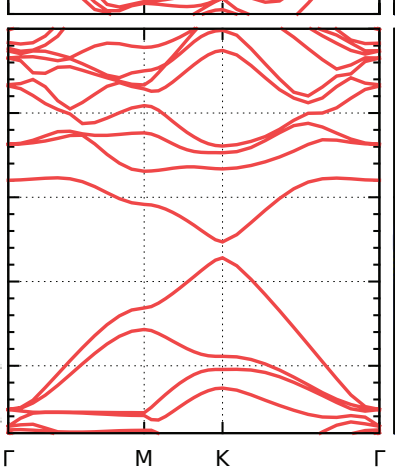

FIG. 3. (Color online) Electronic band dispersions, decomposed density of states (DOS) together with side and top view of the final relaxed structures. (a) Adsorption of $\mathrm{B}$ on valley site, (b) adsorption of $\mathrm{N}$ on bridge site, (c) adsorption of Al on valley site, (d) adsorption of $\mathrm{P}$ on hill site, (e) B, (f) N, (g) Al, and (h) P substituted silicene layers. The energies are relative to the Fermi level $\left(E_{\mathrm{F}}=0\right)$. In the legend, "A" refers to the adsorbed/substituent atom.

contribution originates mainly from the hybridization of $\mathrm{Si}-p_{z}$ and $\mathrm{N}-p_{z}$ bands, tails of $s$ and $p_{x y}$ states of both silicene and $\mathrm{N}$ play a role in the metallic behavior. It is also seen that the linearly crossing $p$ bands of silicene at the $K$ symmetry point are disturbed because of the interaction with the $\mathrm{N}$ $p_{z}$ states albeit not completely removed as for $\mathrm{B}$ adsorption. The electronic band dispersion character of $\mathrm{Al}$ adsorption is qualitatively identical to the one of the B adsorption, caused by the similar adsorption site and valence electron configuration. The last case of $\mathrm{P}$ adsorption shows that the metallic bands are predominantly formed by the hybridization of the P- $p_{x y}$ states with the $\mathrm{Si}-p_{x y}$ and the $\mathrm{Si}-p_{z}$ states and are partially produced from silicene $s$ states. The linear crossing of $p$ bands of silicene at the $K$ symmetry point is again completely removed.

On a graphene lattice, upon $\mathrm{B}$ adsorption, $0.4 e$ is transferred to graphene, and upon $\mathrm{N}$ adsorption, 0.7e is transferred from graphene to $\mathrm{N} .^{38}$ However, due to its $s p^{3}$-like lattice structure, silicene has a highly reactive surface and therefore some different adsorption characteristics can be expected. The charge transfer occurs from silicene to the adsorbate for the 
$\mathrm{B}, \mathrm{N}$, and $\mathrm{P}$ adatoms. The opposite charge transfer is observed for the $\mathrm{Al}$ adatom. The net charge located on $\mathrm{B}, \mathrm{N}, \mathrm{Al}$, and $\mathrm{P}$ adatom has a value of $-1.6,-2.0,1.0$, and $-0.9 e$, respectively. Moreover, in order to examine the reliability of these results, we have also performed a charge population analysis with the iterative modified Hirshfeld method. ${ }^{34,35}$ We found that all the methods provide the same qualitative insight and support for the conclusion that the character of the charge transfer is driven by the differences of electronegativity among the atoms. Silicene donates/accepts electrons to/from adsorbates with higher/smaller electronegativity.

Substitutional doping of silicene induces an opposite change of the work function as compared to adsorption. B or Al substitution increases and $\mathrm{N}$ or $\mathrm{P}$ substitution slightly decreases the work function. The electronic band structure together with the density of states are shown in Figs. 3(e)-3(h). The Dirac cone composed of $p_{z}$ bands is preserved for $\mathrm{B}, \mathrm{Al}$, and $\mathrm{P}$ substitutions. It has been shifted above the Fermi level for $\mathrm{B}$ and $\mathrm{Al}$ substituents and below the Fermi level for a $\mathrm{P}$ atom. It is not altered by the $p_{x y}$ states of the foreign atom, which are present as deep bands in the final electronic structure. The character of substitution with a $\mathrm{N}$ atom is of a different nature, the linear crossing of $p$ bands is destroyed and the metallic bands in the electronic structure are produced from both $p$ and $s$ states located on $\mathrm{N}$ and the surrounding $\mathrm{Si}$ atoms. These states originate from unoccupied states of the free $\mathrm{N}$ atom and align with the Fermi level in doped silicene.

The calculated charge transfers for the substitutional doping are of the same magnitude as for adsorption, with 1.4, 2.1, -1.6 , and $1.2 e$ transferred from silicene to a $\mathrm{B}, \mathrm{N}, \mathrm{Al}$, and $\mathrm{P}$ atoms, respectively. Only $\mathrm{N}$-doped silicene becomes ferromagnetic with a net magnetic moment of $0.9 \mu_{\mathrm{B}}$.

The size of the gap as well as the character of the electronic dispersion bands may be altered by the adsorption or substitution of foreign atoms with the applied spin-orbit interaction as in the case of graphene decorated by $5 d$ transition-metal adatoms. ${ }^{39}$ We performed calculations with spin-orbit coupling included in order to investigate its effect in all the studied systems and found no significant differences in the electronic band dispersion. The variances were found to be in the order of $1 \mathrm{meV}$ and virtually vanishing around the Fermi level. Therefore we do not report these results in Fig. 3. The cause of such negligible coupling is the small diameter of the adsorbate/substituent atoms and the small or not existing net magnetic moment of the aforementioned structures. $^{40}$

\section{Stability analysis}

Characterization and analysis of the surfaces, crystal structures and molecules can be done by various spectroscopy techniques. Among these particularly Raman spectroscopy allows highly accurate detection and analysis of the vibrational properties of structures even at the nanoscale. Therefore one can deduce the stability and characteristic vibrational properties of a foreign atom adsorbed/substituted silicene layers via their phonon spectrum. Figure 4 shows the evolution of the phonon density of states of pristine silicene with adsorption and substitution of $\mathrm{B}, \mathrm{N}, \mathrm{Al}$, and $\mathrm{P}$ atoms. Pristine silicene's phonon DOS comprises three main peaks originating
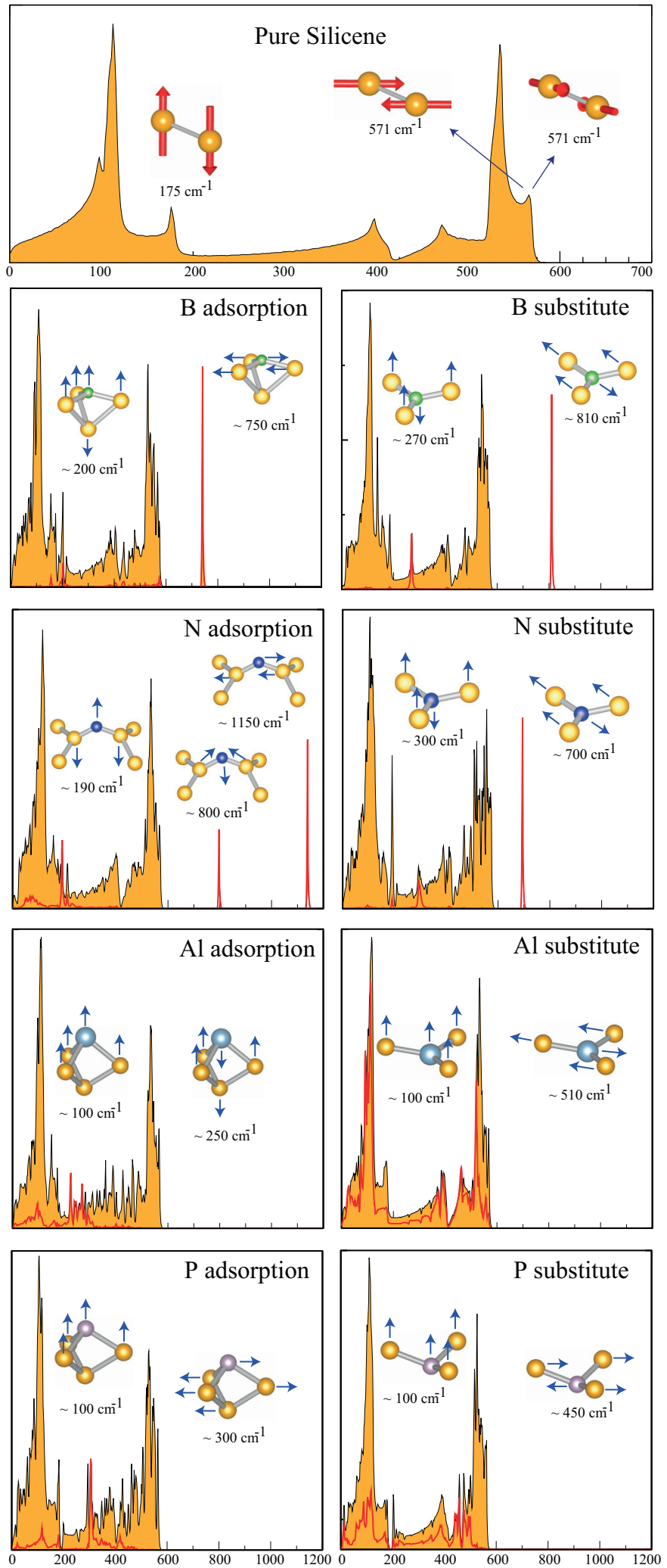

Frequency $\left(\mathrm{cm}^{-1}\right)$

FIG. 4. (Color online) Phonon dispersion for adsorption and substitution cases of $\mathrm{B}, \mathrm{N}, \mathrm{Al}$ and $\mathrm{P}$ on silicene. Total DOS of the structures is shown by the filled area (orange color). Projected DOS belonging to foreign atoms are represented by lines (red). $\Gamma$ point vibrational motions are also depicted. 
(a)

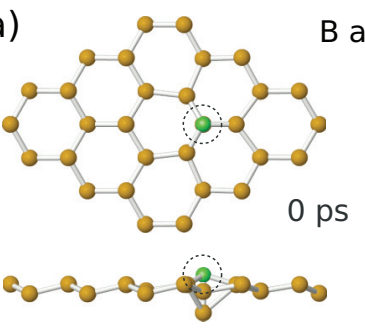

B adsorption

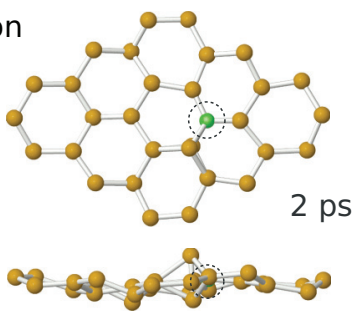

(e)

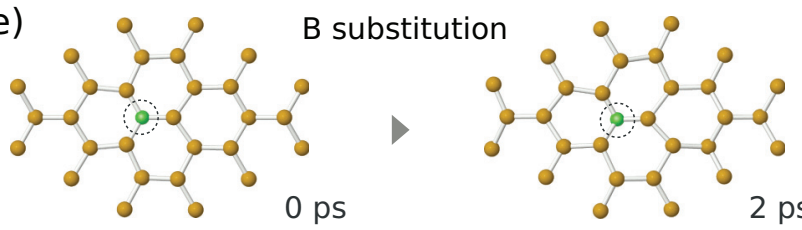

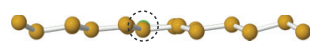

820050300 (b)

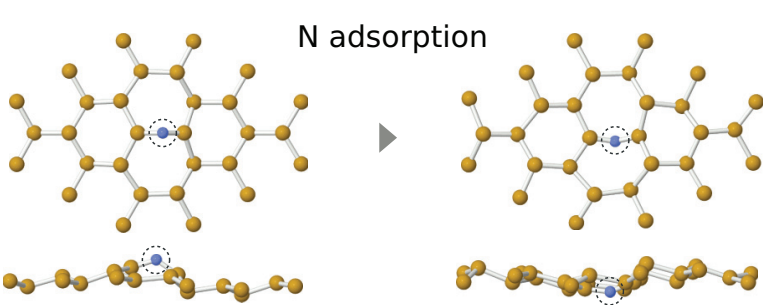

(c)

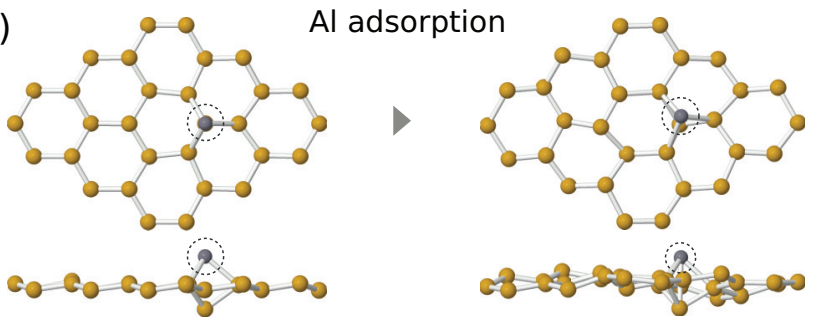

(d)

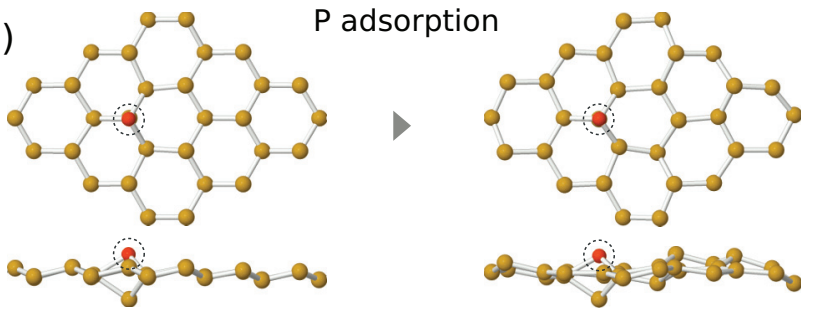

(f)

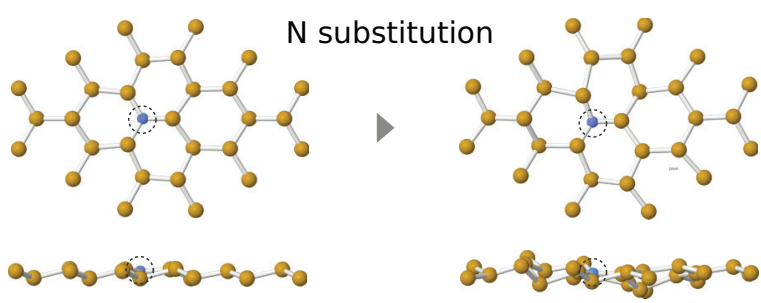

(g)

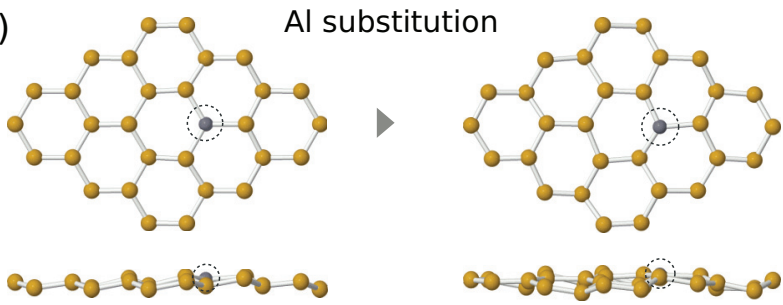

(h)

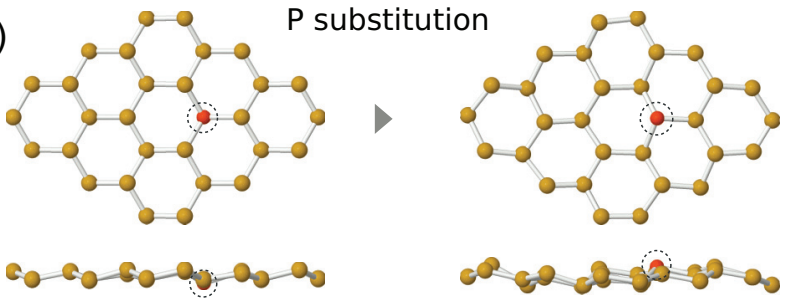

FIG. 5. (Color online) Top and side view of the ground-state geometry (left) and the changed geometry at $T=500 \mathrm{~K}$ after 2 ps of molecular dynamics (right). The adsorbate/substituent atoms are marked with an open dotted circle for clarity.

from the $\Gamma$ atomic motions: an acoustic mode at $\sim 100 \mathrm{~cm}^{-1}$, an out-of-plane optical mode at $\sim 175 \mathrm{~cm}^{-1}$, and a degenerate in-plane optical mode at $\sim 571 \mathrm{~cm}^{-1}$. Note that the peaks at 396,472 , and $535 \mathrm{~cm}^{-1}$, are due to the flat slope of the optical branch at the $M-K, M$, and $M$ symmetry points, respectively. We also present adsorbate/substituent-projected phonon DOS and related vibrational motions at the $\Gamma$ point in Fig. 4.

Adsorption/substitution of first-row elements $\mathrm{B}$ and $\mathrm{N}$ do not result in a significant change of the DOS. Due to the coupling of $\mathrm{B}$ and $\mathrm{N}$ adsorbates/substituents to the acoustical phonon branch of pristine silicene, several sharp peaks appear between 200 and $300 \mathrm{~cm}^{-1}$. Obviously, among the two adsorbates, $\mathrm{B}$ and $\mathrm{N}$, the bridge-site-bonded $\mathrm{N}$ adatom is more likely to mix with silicene's acoustic branch. In addition to these, high-frequency adsorbate/substituent induced modes appear between 700 and $1200 \mathrm{~cm}^{-1}$. Our eigenvector analysis reveals that these high energy modes correspond to in-plane bond-stretching motion of the adatom and the neighboring silicon atoms.

Consistent with the binding energies calculated in the previous section, the high-frequency in-plane phonon mode of B-substituted silicene takes a higher value than that of the $\mathrm{B}$-adsorbed one. However, such a comparison is not possible for the $\mathrm{N}$ atom because of the different geometries of the adsorption and substitution cases. Since the adsorption of a $\mathrm{N}$ atom occurs on the bridge site of two silicon atoms, one can expect vibrational characteristics different from those of $\mathrm{N}$-doped graphene. While there is no clear $\mathrm{N}$-induced peak observed in graphene, upon adsorption (substitution) of $\mathrm{N}$ to silicene, well separated phonon modes (mode) at $\sim 800 \mathrm{~cm}^{-1}$ and $\sim 1150$ (only at 700 ) $\mathrm{cm}^{-1}$ appear. Furthermore, compared with $\mathrm{B}$ substitution, $\mathrm{N}$ substituted silicene's high-frequency mode is softer because of the larger atomic mass of $\mathrm{N}$. These modes correspond to in-plane and out-of-plane motion of the $\mathrm{N}$ and the nearest two $\mathrm{Si}$ atoms. Contributions of second and third nearest-neighbor $\mathrm{Si}$ atoms are negligible for these high-frequency modes. Because of their bond-stretching nature, adsorbate- and substituent-originated optical branches can be expected to be Raman-active modes.

The experimentally reported atomic weights (covalent atomic radii) of $\mathrm{Si}, \mathrm{B}, \mathrm{N}, \mathrm{Al}$, and $\mathrm{P}$ are $28.09,10.81,14.01$, 26.98, and $30.97 \mathrm{amu}(111,84,71,121$, and $107 \mathrm{pm})$ and 
therefore due to the similar properties of second row elements to $\mathrm{Si}$ atoms, for the adsorption/substitution of $\mathrm{Al}$ and $\mathrm{P}$ atoms different effects on the vibrational spectra can be expected. We report a common characteristic for second row elements, that is the absence of high-frequency bond stretching modes. Due to the quite similar atomic weight and radii of $\mathrm{Si}, \mathrm{Al}$, and $\mathrm{P}$ atoms, their effect on the phonon DOS is almost negligible and may not be observed by experimental tools. A widely broadened phonon DOS of $\mathrm{Al}$ and $\mathrm{P}$ adsorbed silicene, which is different from the narrow peaks in the $\mathrm{B}$ and $\mathrm{N}$ adsorption case, indicates the larger coupling of $\mathrm{Al}$ atoms to the silicene lattice. Similarly, the substitution of $\mathrm{Al}$ and $\mathrm{P}$ does not result in the presence of high energy modes. It is also seen that while substituent $\mathrm{B}$ and $\mathrm{N}$ atoms do not couple with optical modes of silicene, $\mathrm{Al}$ and $\mathrm{P}$ atoms entirely contribute to both acoustic and optical modes.

The discussed structures that are found to be stable in terms of the total energy optimization and phonon calculations may not be stable at high temperatures. To address questions regarding thermal stability of a B and $\mathrm{N}$ atom adsorbed/substituted silicene monolayers, we consider the effect of temperature by employing ab initio MD calculations. MD simulations were performed at a temperature of $500 \mathrm{~K}$ for the structures initially optimized at $T=0 \mathrm{~K}$.

In Fig. 5, geometric structures are presented for the initial and final states of the MD calculations. The simulation of B adsorption, as can be seen in Fig. 5(a), shows that the B atom stays incorporated within the honeycomb structure but the $\mathrm{Si}$ atom, that has been initially pushed down from its position, has started to move towards the neighboring sites. During the $2 \mathrm{ps}$ of MD simulations, it is attached predominantly on the hollow and the top sites. However, the adsorbed B atom remains stable. Similarly, the substituent B atom [see Fig. 5(e)] continues to be bonded to the three $\mathrm{Si}$ atoms for the whole simulation period.

Although the adsorption geometry for the $\mathrm{N}$ atom is different from B-adsorbed silicene, it remains stable after 2 ps at $500 \mathrm{~K}$. Even the characteristic out-of-plane motion of the $\mathrm{N}$ atom (from one side to the other one), that can be clearly seen in Fig. 5(b), is not able to break the strong $\mathrm{Si}-\mathrm{N}-\mathrm{Si}$ bonds. Similar to the B substitution case, the N-substituted silicene, depicted in Fig. 5(f), remains in-plane bonded to the three neighboring silicon atoms after more than 2 ps at $500 \mathrm{~K}$.

Simulation of $\mathrm{Al}$ and $\mathrm{P}$ adsorption [see Figs. 5(c) and 5(d)] as well as $\mathrm{Al}$ and $\mathrm{P}$ substitution [see Figs. 5(g) and 5(h)] shows high stability of the adsorbate/substituent atom with no reconfiguration of the Si lattice in the vicinity of the foreign atoms.

\section{CONCLUSIONS}

In summary, we performed an $a b$ initio study of atom adsorption and absorption of $\mathrm{B}, \mathrm{N}, \mathrm{Al}$, and $\mathrm{P}$ atoms on silicene, and we obtained the geometric, electronic, and vibrational properties. All the final systems show metallic behavior. The adatoms are strongly bonded with silicene revealing different preferential adsorption sites for $\mathrm{B}$ or $\mathrm{N}$ adatoms as compared to graphene. We find the charge transfer to be consistent with the effects ascribed to the element electronegativity. $\mathrm{B}, \mathrm{N}$, and $\mathrm{P}$ atoms behave like acceptors and the $\mathrm{Al}$ atom as donor for silicene. Analysis of the vibrational modes shows that adatoms and substituents slightly alter the phonon spectrum of silicene and several adatom/substituent-induced characteristic branches appear, which may be probed by Raman measurements. Finally, we showed the stability of $\mathrm{B}, \mathrm{N}, \mathrm{Al}$, and $\mathrm{P}$ adsorbed/substituted silicene layers even at high temperatures. Our work reveals that silicene has a very reactive and functionalizable surface and it can serve as an important and novel playground for silicene based novel nanoscale materials.

\section{ACKNOWLEDGMENTS}

This work was supported by the Flemish Science Foundation (FWO-V1) and the Methusalem foundation of the Flemish government. Computational resources were provided by TUBITAK ULAKBIM, High Performance and Grid Computing Center (TR-Grid e-Infrastructure), and HPC infrastructure of the University of Antwerp (CalcUA) a division of the Flemish Supercomputer Center (VSC), which is funded by the Hercules foundation. H.S. is supported by a FWO Pegasus Marie Curie Fellowship.

\footnotetext{
*jozef.sivek@ua.ac.be

†hasan.sahin@ua.ac.be

†bart.partoens@ua.ac.be

§rancois.peeters@ua.ac.be

${ }^{1}$ K. S. Novoselov, A. K. Geim, S. V. Morozov, D. Jiang, Y. Zhang, S. V. Dubonos, I. V. Grigorieva, and A. A. Firsov, Science 306, 666 (2004).

${ }^{2}$ A. K. Geim and K. S. Novoselov, Nat. Mater. 6, 183 (2007).

${ }^{3}$ D. C. Elias, R. R. Nair, T. M. G. Mohiuddin, S. V. Morozov,

P. Blake, M. P. Halsall, A. C. Ferrari, D. W. Boukhvalov, M. I.

Katsnelson, A. K. Geim et al., Science 323, 610 (2009).

${ }^{4}$ R. R. Nair, W. Ren, R. Jalil, I. Riaz, V. G. Kravets, L. Britnell, P. Blake, F. Schedin, A. S. Mayorov, S. Yuan et al., Small 6, 2877 (2010).

${ }^{5}$ H. Şahin, M. Topsakal, and S. Ciraci, Phys. Rev. B 83, 115432 (2011).
}

${ }^{6}$ O. Leenaerts, H. Peelaers, A. D. Hernández-Nieves, B. Partoens, and F. M. Peeters, Phys. Rev. B 82, 195436 (2010).

${ }^{7}$ H. Şahin and S. Ciraci, J. Phys. Chem. C 116, 24075 (2012).

${ }^{8}$ L. S. Panchakarla, K. S. Subrahmanyam, S. K. Saha, A. Govindaraj, H. R. Krishnamurthy, U. V. Waghmare, and C. N. R. Rao, Adv. Mater. (Weinheim, Ger.) 21, 4726 (2009).

${ }^{9}$ Z.-H. Sheng, H.-L. Gao, W.-J. Bao, F.-B. Wang, and X.-H. Xia, J. Mater. Chem. 22, 390 (2012).

${ }^{10}$ Y. Wang, Y. Shao, D. W. Matson, J. Li, and Y. Lin, ACS Nano 4, 1790 (2010).

${ }^{11}$ A. Lherbier, X. Blase, Y.-M. Niquet, F. Triozon, and S. Roche, Phys. Rev. Lett. 101, 036808 (2008).

${ }^{12}$ P. A. Denis, Chem. Phys. Lett. 492, 251 (2010).

${ }^{13}$ K. Takeda and K. Shiraishi, Phys. Rev. B 50, 14916 (1994).

${ }^{14}$ S. Cahangirov, M. Topsakal, E. Aktürk, H. Şahin, and S. Ciraci, Phys. Rev. Lett. 102, 236804 (2009). 
${ }^{15}$ H. Şahin, S. Cahangirov, M. Topsakal, E. Bekaroglu, E. Akturk, R. T. Senger, and S. Ciraci, Phys. Rev. B 80, 155453 (2009).

${ }^{16}$ C.-C. Liu, W. Feng, and Y. Yao, Phys. Rev. Lett. 107, 076802 (2011).

${ }^{17}$ C.-C. Liu, H. Jiang, and Y. Yao, Phys. Rev. B 84, 195430 (2011).

${ }^{18}$ M. Topsakal and S. Ciraci, Phys. Rev. B 81, 024107 (2010).

${ }^{19}$ M. Ezawa, Phys. Rev. Lett. 109, 055502 (2012).

${ }^{20}$ P. D. Padova, C. Quaresima, C. Ottaviani, P. M. Sheverdyaeva, P. Moras, C. Carbone, D. Topwal, B. Olivieri, A. Kara, H. Oughaddou et al., Appl. Phys. Lett. 96, 261905 (2010).

${ }^{21}$ P. D. Padova, C. Leandri, S. Vizzini, C. Quaresima, P. Perfetti, B. Olivieri, H. Oughaddou, B. Aufray, and G. L. Lay, Nano Lett. 8, 2299 (2008).

${ }^{22}$ P. Vogt, P. De Padova, C. Quaresima, J. Avila, E. Frantzeskakis, M. C. Asensio, A. Resta, B. Ealet, and G. Le Lay, Phys. Rev. Lett. 108, 155501 (2012).

${ }^{23}$ C.-L. Lin, R. Arafune, K. Kawahara, N. Tsukahara, E. Minamitani, Y. Kim, N. Takagi, and M. Kawai, Appl. Phys. Express 5, 045802 (2012).

${ }^{24}$ H. Jamgotchian, Y. Colignon, N. Hamzaoui, B. Ealet, J. Y. Hoarau, B. Aufray, and J. P. Bibérian, J. Phys.: Condens. Matter 24, 172001 (2012).

${ }^{25}$ A. Fleurence, R. Friedlein, T. Ozaki, H. Kawai, Y. Wang, and Y. Yamada-Takamura, Phys. Rev. Lett. 108, 245501 (2012).
${ }^{26}$ H. Sahin and F. M. Peeters, Phys. Rev. B 87, 085423 (2013).

${ }^{27}$ M. Oubal, S. Picaud, M.-T. Rayez, and J.-C. Rayez, Comp. Theor. Chem. 990, 159 (2012).

${ }^{28}$ G. Kresse and J. Furthmüller, Phys. Rev. B 54, 11169 (1996).

${ }^{29}$ D. M. Ceperley and B. J. Alder, Phys. Rev. Lett. 45, 566 (1980).

${ }^{30}$ P. E. Blöchl, Phys. Rev. B 50, 17953 (1994).

${ }^{31}$ P. E. Blöchl, O. Jepsen, and O. K. Andersen, Phys. Rev. B 49, 16223 (1994).

${ }^{32}$ R. F. W. Bader, Chem. Rev. (Washington, DC, US) 91, 893 (1991).

${ }^{33}$ G. Henkelman, A. Arnaldsson, and H. Jónsson, Comput. Mater. Sci. 36, 354 (2006).

${ }^{34}$ F. L. Hirshfeld, Theor. Chim. Acta 44, 129 (1977).

${ }^{35}$ P. Bultinck, C. V. Alsenoy, P. W. Ayers, and R. Carbó-Dorca, J. Chem. Phys. 126, 144111 (2007).

${ }^{36}$ D. Alfè, Comput. Phys. Commun. 180, 2622 (2009).

${ }^{37}$ M. Ezawa, J. Phys. Soc. Jpn. 81, 064705 (2012).

${ }^{38} \mathrm{~K}$. Nakada and A. Ishii, in Graphene Simulation, edited by Jian Ru Gong (InTech, Rijeka, Croatia, 2011), Chap. 1.

${ }^{39}$ H. Zhang, C. Lazo, S. Blügel, S. Heinze, and Y. Mokrousov, Phys. Rev. Lett. 108, 056802 (2012).

${ }^{40}$ J. Hu, J. Alicea, R. Wu, and M. Franz, Phys. Rev. Lett. 109, 266801 (2012). 